\title{
BTW - Bioinformatics Through Windows: an easy-to-install package to analyze marker gene data
}

\author{
Daniel Morais ${ }^{1}$, Luiz Roesch ${ }^{2}$, Marc Redmile-Gordon ${ }^{3}$, Fausto Santos ${ }^{4}$, Petr Baldrian ${ }^{1}$, Fernando Andreote \\ 5, Victor S Pylro Corresp. 5, 6 \\ 1 Institute of Microbiology, Czech Academy of Sciences, Prague, Czech Republic \\ 2 Centro para Pesquisa Interdisciplinar em Biotecnologia, Universidade Federal do Pampa, São Gabriel, Rio Grande do Sul, Brazil \\ 3 Natural England, Sheffield, United Kingdom \\ 4 Biosystems Informatics and Genomics, Instituto René Rachou, Belo Horizonte, Brazil \\ 5 Soil Department, "Luiz de Queiroz" College of Agriculture - ESALQ/USP, Piracicaba, São Paulo, Brazil \\ 6 Department of Biology, Federal University of Lavras - UFLA, Lavras, Minas Gerais, Brazil \\ Corresponding Author: Victor S Pylro \\ Email address: victor.pylro@brmicrobiome.org
}

Recent advances in Next-Generation Sequencing (NGS) make comparative analyses of the composition and diversity of whole microbial communities possible at far greater depth than ever before. This brings new challenges, such as an increased dependence on computation to process these huge datasets. The demand on system resources usually requires migrating from Windows to Linux-based operating systems and prior familiarity with command-line interfaces. To overcome this barrier, we developed a fully automated and easy-to-install package as well as a complete, easy to follow pipeline for microbial metataxonomic analysis operating in the Windows Subsystem for Linux (WSL) Bioinformatics Through Windows (BTW). BTW combines several open-access tools for processing marker gene data, including 16S rRNA, bringing the user from raw sequencing reads to diversity-related conclusions. It includes data quality filtering, clustering, taxonomic assignment and further statistical analyses, directly in WSL, avoiding the prior need of migrating from Windows to Linux. BTW is expected to boost the use of NGS amplicon data by facilitating rapid access to a set of bioinformatics tools for Windows users. Moreover, several Linux command line tools became more reachable, which will enhance bioinformatics accessibility to a wider range of researchers and practitioners in the life sciences and medicine. BTW is available in GitHub (https://github.com/vpylro/BTW). The package is freely available for noncommercial users. 


\author{
BTW - Bioinformatics Through Windows: an easy-to-install package to analyze marker gene data \\ Daniel Kumazawa Morais ${ }^{1}$, Luiz Fernando Wurdig Roesch ${ }^{2}$, Marc Redmile-Gordon ${ }^{3}$, Fausto Gonçalves dos Santos ${ }^{4}$, \\ Petr Baldrian$^{1}$, Fernando Dini Andreote ${ }^{5}$, Victor Satler Pylro ${ }^{5,6 *}$ \\ ${ }^{1}$ Institute of Microbiology of the Czech Academy of Sciences, Prague, Czech Republic. \\ ${ }^{2}$ Centro para Pesquisa Interdisciplinar em Biotecnologia, CIP-Biotec, Universidade Federal do Pampa, São Gabriel, Rio Grande do \\ Sul, Brazil. \\ ${ }^{3}$ Natural England, UK. \\ ${ }^{4}$ René Rachou Research Center, FIOCRUZ-MG. Belo Horizonte, MG, 30190-002. Brazil. \\ ${ }^{5}$ Department of Soil Science, "Luiz de Queiroz" College of Agriculture, ESALQ/USP. Piracicaba, São Paulo, Brazil. \\ ${ }^{6}$ Department of Biology, "Federal University of Lavras. Lavras, Minas Gerais, Brazil. \\ *To whom correspondence should be addressed: victor.pylro@brmicrobiome.org
}

\title{
Abstract
}

Recent advances in Next-Generation Sequencing (NGS) make comparative analyses of the composition and diversity of whole microbial communities possible at far greater depth than ever before. This brings new challenges, such as an increased dependence on computation to process these huge datasets. The demand on system resources usually requires migrating from Windows to Linux-based operating systems and prior familiarity with command-line interfaces. To overcome this barrier, we developed a fully automated and easy-to-install package as well as a complete, easy to follow pipeline for microbial metataxonomic analysis operating in the Windows Subsystem for Linux (WSL) - Bioinformatics Through Windows (BTW). BTW combines several open-access tools for processing marker gene data, including $16 \mathrm{~S}$ rRNA, bringing the user from raw sequencing reads to diversity-related conclusions. It includes data quality filtering, clustering, taxonomic assignment and further statistical analyses, directly in WSL, avoiding the prior need of migrating from Windows to Linux. BTW is expected to boost the use of NGS amplicon data by facilitating rapid access to a set of bioinformatics tools for Windows users. Moreover, several Linux command line tools became more reachable, which will enhance bioinformatics accessibility to a wider range of researchers and practitioners in the life sciences and medicine. BTW is available in GitHub (https://github.com/vpylro/BTW). The package is freely available for noncommercial users.

\section{Introduction}

In April 2016, Microsoft announced the release of the Windows Sub-system for Linux (WSL), which is available to Windows 10 users. This distribution consists of a Linux environment compiled through Windows and enables most native command-line tools, utilities and binaries from Linux to run on Windows: the users can now run Bash scripts and all popular Linux command-line tools like sed, awk, grep, sort, apt, ssh and others. One anticipated outcome was that this effort would bring free software to a wider audience, since Windows is the native Operating System (OS) in $\sim 85 \%$ of the Desktops and Laptops worldwide (per StatCounter for June 2017 -

41 http://gs.statcounter.com/os-market-share/desktop/worldwide). However, a further but perhaps unconsidered benefit of expanding bioinformatics accessibility, is that Linux command line tools can be used to run several bioinformatics applications on the hardware available without the need for a dedicated ma-chine, or the hassle of having multiple operating systems on a single machine (dual-boot or virtual machines). 
Biologists have recently entered the world of big data (Marx, 2013), consisting of cross-referenced databases, ranging from DNA to metabolic pathways (Cook et al., 2015). However, it is still challenging to effectively perform data analyses using these databases and to manipulate high throughput sequencing data. This is largely because bioinformatics software is typically developed for Unix Shell (Seemann, 2013), and mastery of its command-line interface usually requires intensive training. The command-line interface allows users to easily store and document all the steps taken during the data analysis. This can be achieved through the creation of scripts (chronologically organized list of commands connected by their inputs and outputs) that can be executed thoroughly with one line of code in the command-line interface. This helps to automatize repetitive work and facilitates reproducibility. As previously defined by Mushegian (2011), bioinformatics deals with a dual existence paradigm: as developing technology (the tools), and as a science that applies these tools. In the former, providing a user-friendly graphic interface for data analysis is not always feasible even though command-line gives users and developers flexibility to manipulate and sort data. Attempts have been made to help researchers outside the bioinformatics discipline to use tools dedicated to sequence analysis, for instance, MG-RAST (Meyer et al., 2008), SEED2 (Vetrovsky et al., 2018) and BMP desktop (Pylro et al., 2016). Even so, bioinformatics has been the cause of headache for many scientists, even for those who grew up in the computer era.

Making bioinformatics accessible to everyone has been one of the main challenges of contemporary biology. Through the development of bioinformatics tools and training of users in biological data assessment, the Brazilian Microbiome Project (BMP http://brmicrobiome.org - Pylro et al., 2014a) and the Center for Systems Biology (http://c4sys.cz), we observed both students and adept professionals in biological sciences struggle when facing the command-line interfaces of the Unix-based OS (such as Linux and macOSX). The new WSL-Ubuntu feature presented here is aimed to help biologists to access Next-Generation Sequencing (NGS) analysis tools without the above limitations. Although the native Bash tools present at Ubuntu operating systems are useful for manipulating biological data, this distribution doesn't come without specific bioinformatics packages, which require several steps of settings and installation before usage. To overcome this issue, we have created an easy to follow tutorial to installing WSL (available on http://brmicrobiome.org/tutorialbtw) and a Bash script (freely available on https://github.com/vpylro/BTW) that should be run through the command-line of WSL-Ubuntu, to set up all the necessary packages for running basic NGS data manipulations and the full microbial community metataxonomic analysis, as previously provided by the BMP to UNIX-based operating systems users (Pylro et al., 2014b). We also created a benchmark comparing the performance of the metataxonomic pipeline under the WSL-Ubuntu 16.04 with pure Ubuntu 16.04 distribution and using an Ubuntu as a Virtual Machine inside the Windows 10 platform.

\section{Methods}

To demonstrate the functionality and performance of WSL-Ubuntu in running a complete 16S rRNA data analysis pipeline, we assessed the operation of Qiime 1.9 (Caporaso et al., 2010), VSEARCH 2.4.4 (Rognes et al., 2016) and BMP Scripts (Pylro et al., 2014b). This bioinformatics pipeline is used to describe and compare the

81 prokaryotic composition in a group of samples. It relies on the high conservation degree and widespread presence of 
82 the $16 \mathrm{~S}$ gene in prokaryotes. Using high-throughput sequencing technologies to sequence the product of a PCR

83 (polymerase chain reaction), generated with universal primers designed to amplify conserved regions of the 16S gene

84 from a DNA sample, millions of DNA sequences will be generated. After treating the sequences to remove sequencing 85 noise and errors, we quantify and assign taxonomy to every sequence to have an estimate of the whole prokaryotic community in our samples. Further details of the pipeline for metataxonomic analyses and installation of the software are in the supplementary material (PDF). We found that except for packages that require a graphical display (e.g. core_diversity.py from QIIME), all of them work just as in the pure Ubuntu installation. Programs with graphic output are not yet officially supported by WSL, but from our tests with Xming (http://straightrunning.com/XmingNotes) all the programs performed well. The complete pipeline for 16S rRNA data analysis on WSL is available on http://brmicrobiome.org/win16s (Figure 1). Briefly, 16S reads data of both forward and reverse amplicons are merged into contigs using the "fastq-join" method (Aronesty, 2013) in QIIME. The output file (.fastq) is then quality filtered, trimmed to equal lengths, dereplicated, sorted and binned into operational taxonomic units (OTUs) using VSEARCH commands (Rognes et al., 2016). Taxonomy is assigned to each representative sequence using the RDPclassifier (ribosomal database project) (Wang et al., 2007) against the GreenGenes (13_8) reference database. An OTU Table (biom format) containing both OTU abundance and taxonomy is constructed using QIIME. Finally, the .biom OTU table is fully compatible with the MicrobiomeAnalyst (Dhariwal et al., 2017), a user-friendly web-based platform for microbiome data analyses and visualizations, including taxonomy plots and estimates of $\alpha$ - and $\beta$-diversity (http://microbiomeanalyst.ca).

\section{Results}

Benchmarking

To evaluate the usability of such tool for 16S rRNA amplicon data analysis (Supplemental File 1), we performed a benchmark test using 12,638,185 reads of 16S rRNA gene data generated from paired-end $150 \mathrm{bp} \mathrm{Illumina}$ sequencing (available at https://www.ncbi.nlm.nih.gov/bioproject/241041), in 3 different scenarios: (1) using the BTW package with the WSL in a Windows 10 machine; (2) using a virtual machine (VirtualBox v. 5.2.6 - Oracle) inside a Windows 10 containing Linux Ubuntu 16.04 (BMP OS); and (3) using a dual boot system splitting the hard drive between two operating systems, Windows 10 and Linux Ubuntu 16.04, giving Ubuntu 16.04 full access to processing and RAM memory resources. All tests were performed in a Desktop computer with an Intel ${ }^{\circledR}$ Core ${ }^{\mathrm{TM}}$ i7-

110 M640 (2 cores; 4 logical processors - $2.8 \mathrm{GHz}$ ) and $4 \mathrm{~GB}$ of RAM memory. The time elapsed for processing the same

111 data set in each scenario was 3:09 hours for the WSL-Ubuntu/BTW, 4:14 hours using a virtual machine and 1:27

112 hours in a native Linux Ubuntu 16.04. The outputs were all the same, resulting in a final OTU table in the BIOM 113 format.

\section{Discussion}

116 Our results show different performances among the tree tested approaches. Virtual Machine took the longest 117 to execute the task, in reason of its nature, requiring complete access to part of the computer's hardware (Goldberg 118 1974), splitting the processing power by two Operating Systems. The WSL-Ubuntu/BTW was faster than the virtual 
119 machine, but still took more than twice the time to execute the task, compared to the plain Ubuntu installation. This

120 is related to two characteristics of the WSL-Ubuntu System: (1) WSL has to translate various Linux file system

121 operations into the Windows file system structure and to do so, it creates an extra layer of processing to read and write

122 files, making the process slower (the details of this architecture can be read at the Microsoft WSL support blog:

123 https://blogs.msdn.microsoft.com/wsl/2016/06/15/wsl-file-system-support/), and (2) it might be related to the

124 hardware being busy with Windows and Ubuntu basic processes. The plain Ubuntu 16.04 System performed the

125 fastest, without having to share computing resources neither having extra layers for file system manipulation.

126 Although the pure Ubuntu installation is still the fastest approach, this results show the advantage of using WSL-

127 Ubuntu with the BTW installation, in reason of the users having a practical and immediate access to the Unix-style

128 command-line without having to completely change the operating system or suffer from limitations as splitting their

129 hardware or connecting folders from the host and guest operating systems.

130 Despite being used for 16S rRNA gene NGS data analysis, there is no limitation in using BTW for 18S rRNA

131 and ITS amplicon analyses as well. One concern about the WSL adoption by the scientific community is that this

132 feature is being distributed by a commercial company, who may stop supporting it at any time., On the other hand, if

133 developers, scientists and general users show support through uptake, Microsoft may continue to develop and support

134 the feature. In such an event, it is likely that developers of existing tools currently incompatible to WSL will be

135 convinced to join the movement. WSL is still in the beta phase, meaning that some scripts and tools currently used for

136 bioinformatics will not work perfectly. For instance, we experienced fails [segmentation fault (core dumped)] while

137 running QIIME Uclust-based commands and the USEARCH package (Edgar, 2010) which seems to be an issue related

138 to the compilation process. To solve the mentioned problem, we adopted similar software to perform the same task,

139 in this case VSEARCH (Rognes et al., 2016). Moreover, the bioinformatics users who already learned through this

140 platform, would be able to migrate their knowledge to any full Unix operating system, but without the disadvantage

141 of having to change completely the operating system or use solutions that split their machine's processing power.

\section{Conclusion}

144 BTW has proved useful to facilitate rapid access to bioinformatics resources by Windows users, which will 145 boost analytical capacity for NGS data.

\section{References}

148 Aronesty E. 2013. Comparison of sequencing utility programs. The Open Bioinformatics Journal, 7:1-8.

149 Caporaso JG, Kuczynski J, Stombaugh J, Bittinger K, Bushman FD, Costello EK, Fierer N, Peña AG, Goodrich JK, 150 Gordon JI, Huttley GA, Kelley ST, Knights D, Koenig JE, Ley RE, Lozupone CA, McDonald D, Muegge BD, 151 Pirrung M, Reeder J, Sevinsky JR, Turnbaugh PJ, Walters WA, Widmann J, Yatsunenko T, Zaneveld J, Knight R. 152 QIIME allows analysis of high-throughput community sequencing data. Nat Methods. 7(5):335-336, 2010. doi: 153 10.1038/nmeth.f.303..

154 Cook CE, Bergman MT, Finn RD, Cochrane G, Birney, E, Apweiler R. 2015. The European Bioinformatics Institute 155 in 2016: data growth and integration. Nucleic Acids Res, 44(D1), D20-D26. 
156 Dhariwal A, Chong J, Habib S, King IL, Agellon LB, Xia J. 2017. MicrobiomeAnalyst - a web-based tool for comprehensive statistical, visual and meta-analysis of microbiome data. Nucleic Acids Res, 45, W180-188.

158 Edgar RC. 2010. Search and clustering orders of magnitude faster than BLAST. Bioinformatics, 26,2460 - 2461.

159 Goldberg, RP. 1974. Survey of virtual machine research. Computer, 7, 34-45.

160 Marx V. 2013. Biology: The big challenges of big data. Nature, 498(7453), 255-260.

161 Meyer F, Paarmann D, D'Souza M, Olson R, Glass EM, Kubal M, Paczian T, Rodriguez A, Stevens R, Wilke A, 162 Wilkening J, Edwards RA. 2008. The metagenomics RAST server - a public resource for the automatic 163 phylogenetic and functional analysis of metagenomes. BMC Bioinformatics, 9, 386.

164 Mushegian A. 2011. Grand Challenges in Bioinformatics and Computational Biology. Front Genet, $2,60$.

165 Pylro VS, Morais DK, de Oliveira FS, Santos FG, Lemos LN, Oliveira G, Roesch LF. 2016. BMPOS: A flexible and 166 user-friendly tool sets for microbiome studies. Microb Ecol, 72(2), 443-447.

167 Pylro VS, Roesch LFW, Morais DK, Clark IM, Hirsch PR, Tótola MR. 2014b. Data analysis for 16S microbial 168 profiling from different benchtop sequencing platforms. J Microbiol Methods, 107, 30-37.

169 Pylro VS, Roesch LFW, Ortega JM, Amaral AM, Tótola MR, Hirsch PR, Rosado AS, Góes-Neto A, da Costa da Silva 170 AL, Rosa CA, Morais DK, Andreote FD, Duarte GF, de Melo IS, Seldin L, Lambais MR, Hungria M, Peixoto RS, 171 Kruger RH, Tsai SM, Azevedo V; Brazilian Microbiome Project Organization Committee. 2014a. Brazilian 172 microbiome project: revealing the unexplored microbial diversity—challenges and prospects. Microb Ecol, 67(2), $173 \quad 237-241$.

174 Rognes T, Flouri T, Nichols B, Quince C, Mahé F. 2016. VSEARCH: a versatile open source tool for metagenomics. 175 PeerJ, 4:e2584.

176 Seemann T. 2013. Ten recommendations for creating usable bioinformatics command line software. GigaScience, $1772(1), 15$.

178 Vetrovsky T, Baldrian P, Morais D. 2018. SEED 2: a user-friendly platform for amplicon high-throughput sequencing 179 data analyses. Bioinformatics, bty071.

180 Wang Q, Garrity GM, Tiedje JM, Cole JR. 2007. Naive Bayesian classifier for rapid assignment of rRNA sequences 181 into the new bacterial taxonomy. Applied and environmental microbiology, 73(16), 5261-5267. 


\section{Figure 1}

Flowchart demonstrating the 16S rRNA profiling data analysis pipeline on Windows (WSL).

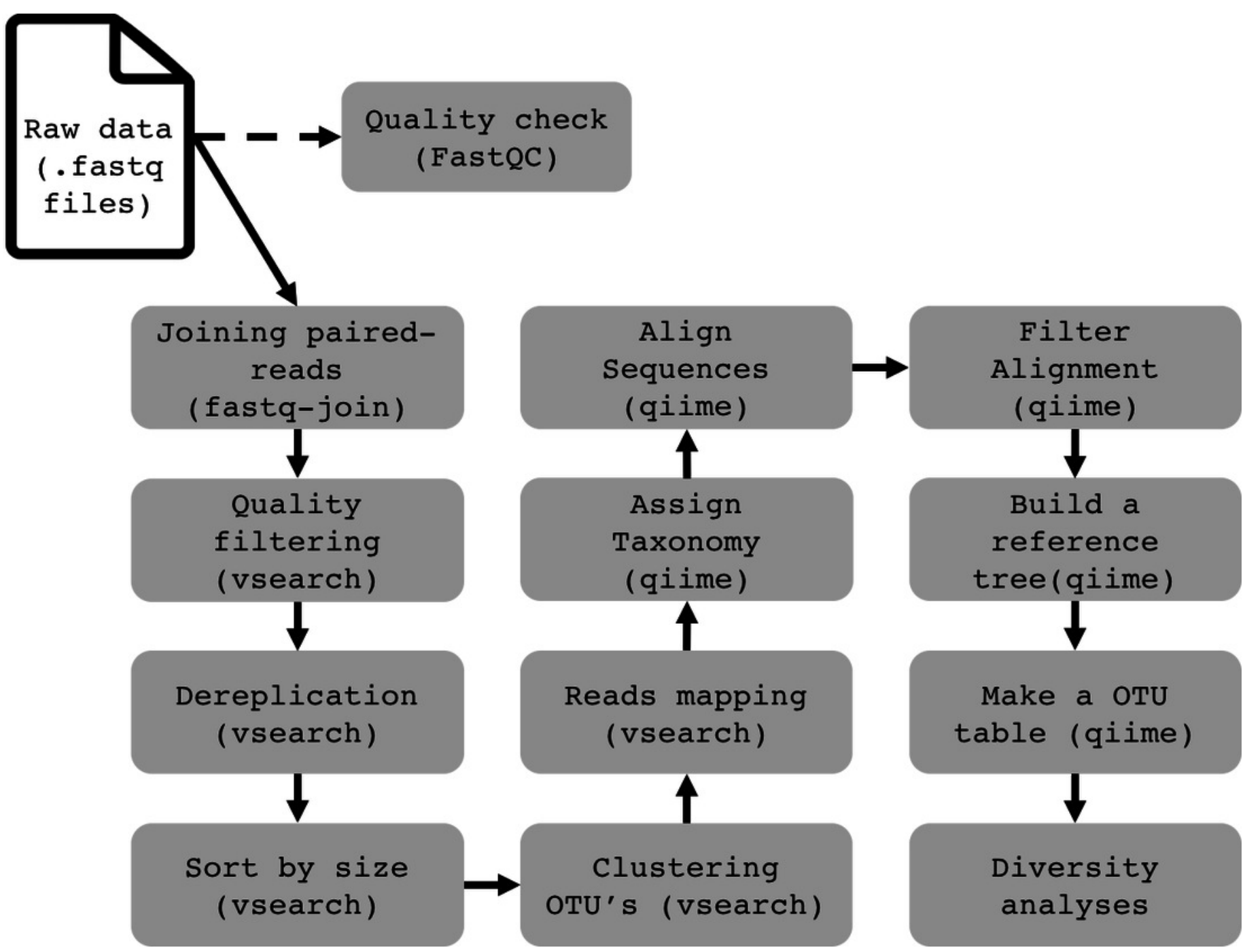

